\title{
Mototaxismo y hurto a personas: evaluación de impacto a partir del modelo de diferencias en diferencias ${ }^{\star}$
}

\author{
Óscar Hernán Cerquera Losada ${ }^{a}$ Cristian Felipe Orjuela Yacueb \\ - Sebastián Esteban Ferraric
}

\begin{abstract}
Resumen: Este trabajo analiza la efectividad de las políticas, adoptadas por las principales ciudades capitales de Colombia para controlar la circulación de motocicletas con pasajero, sobre el hurto a personas durante el periodo 2012-2017. La metodología desarrollada consiste en la aplicación de un modelo de diferencias en diferencias, utilizado generalmente para evaluar el impacto de políticas, mediante la aplicación de un modelo de panel de datos, tanto de efectos fijos como aleatorios; además, se estimó un modelo de regresión lineal múltiple agrupado. Los resultados obtenidos muestran que las políticas adoptadas por estas ciudades han sido efectivas para disminuir el hurto a personas.
\end{abstract}

Palabras clave: Efectos aleatorios; efectos fijos; hurto a personas; mototaxismo.

Fecha de recibido: 23 de noviembre de 2018 Fecha de aprobado: 22 de mayo de 2019

Cómo citar: Cerquera Lozada, Ó. H., Orjuela Yacue, C. F. y Ferrari, S. E. (2019). Mototaxismo y hurto a personas: evaluación de impacto a partir del modelo de diferencias en diferencias. Revista Facultad de Ciencias Económicas, 27(2), 33-50. rev.fac.cienc.econ. doi: https://doi.org/10.18359/rfce.3788

JEL: $C 33,078$.

* Este artículo de investigación se deriva del proyecto de investigación Mototaxismo y hurto en Colombia, del grupo de investigación Iguaque de la Universidad Surcolombiana. Este proyecto no contó con financiación económica por parte de la Universidad, pero sí con dedicación de tiempo por parte de los docentes investigadores.

a Magíster en Economía de la Universidad de Buenos Aires, Argentina. Docente de tiempo completo de planta del programa de Economía de la Universidad Surcolombiana e integrante del grupo de investigación Iguaque. Correo electrónico: oscar.cerquera@usco.edu.co

b Magister en Economía de la Universidad Nacional de la Plata, Argentina. Docente de tiempo completo ocasional del programa de Economía de la Universidad Surcolombiana. Correo electrónico: cristian.orjuela@usco.edu.co

c Magíster en Economía de la Universidad de Buenos Aires, Argentina. Economista sénior de la Cámara Argentina de Comercio y Servicios, y docente semiexclusivo de la Universidad Nacional de La Matanza, Argentina. Correo electrónico: sferrari@cac.com.ar 


\title{
Taxis and Robbery against People: Impact Assessment based on the Differences-In-Differences Model
}

\begin{abstract}
This paper analyzes the effectiveness of policies adopted by the main capital cities of Colombia to control the movement of motorcycles with passenger seats, concerning robbery against people from 2012 to 2017. The developed methodology applies a differences-in-differences model generally used to assess the impact of policies through the implementation of a data-panel model for both fixed and random effects; moreover, a multiple, clustered, linear regression model was also considered. The findings show that the policies adopted by these cities have been effective to reduce robbery against people.
\end{abstract}

Keywords: Random effects; fixed effects; robbery against people; motorcycle taxi.

\section{Mototáxis e roubo de pessoas: avaliação de impacto a partir do modelo de diferenças em diferenças}

Resumo: Este artigo analisa a eficácia das políticas, adotadas pelas principais capitais da Colômbia, para controlar o tráfego de motocicletas com passageiros durante a problemática de roubo a pedestres, entre 2012 e 2017. A metodologia desenvolvida consiste na aplicação de um modelo de diferenças em diferenças, geralmente usado para avaliar o impacto das políticas, através da aplicação de um modelo de painel de dados, tanto de efeitos fixos quanto aleatórios. Além disso, um modelo de regressão linear múltipla agrupada foi estimado. Os resultados obtidos mostram que as políticas adotadas por esses municípios têm sido eficazes na redução do roubo a pessoas.

Palavras-chave: Efeitos aleatórios; efeitos fixos; roubo a pessoas; mototáxis; motorcycle. 


\section{Introducción}

El mototaxismo es un problema social que tiene su origen ante todo en la forma como está estructurada la prestación del servicio de transporte público y más importante en el modelo económico colombiano (Ardilla, 2006).

El sector informal urbano es definido en la decimoquinta Conferencia Internacional de Estadísticos del Trabajo (CIET) (1993) como un conjunto de unidades dedicadas a la producción de bienes o la prestación de servicios, con la finalidad primordial de crear empleos y generar ingresos para las personas que participan en esa actividad.

De acuerdo con la Organización Internacional del Trabajo (отт) (2013a), el mototaxismo surge en Colombia hace más de una década por múltiples factores, entre los cuales se destaca el alto índice de desempleo, la mala calidad del transporte público y la necesidad de generar ingresos. Algunos autores (Arboleda Lozano y Cobo Cartagena, 2016; Espinosa Gutiérrez, Rodríguez Higuera y Uribe Fernández, 2008) coinciden en afirmar que el mototaxismo tuvo sus orígenes en Santa Cruz de Lorica en el corregimiento de Mata de Caña, departamento de Córdoba, y se extendió por todos los corregimientos vecinos hacia la década de los 80. Surgió por la necesidad que tenían los individuos de estas poblaciones de desplazarse hasta la carretera que comunica a Montería y al casco urbano de la localidad.

Lo cierto es que el mototaxismo, considerada una actividad ilegal por parte del Gobierno nacional, ha traído diferentes consecuencias, dependiendo del contexto donde se interprete, y ha generado un gran problema socioeconómico, pues son muchas las personas que consiguen con este trabajo el ingreso para satisfacer sus necesidades. No obstante, en los últimos años han surgido organizaciones o empresas estructuradas de manera informal que se han adueñado del mercado. Igualmente, si existe una oferta de este servicio es porque también hay una fuerte y creciente demanda, motivada por el servicio útil o porque no tiene acceso a otros medios de transporte.

El costo de oportunidad entre la formalidad y la informalidad en países como Colombia, donde una de cada dos personas ingresa a la educación superior ${ }^{1}$, parece favorecer a los últimos, salvo en algunos casos con características especiales. El ingreso obtenido por un mototaxista propietario puede llegar a ser más alto que el ingreso conseguido por ese mismo individuo en otras actividades económicas formales o informales. Aun cuando para ser propietario es necesaria la compra de una moto que implica una alta inversión, el retorno de esta actividad es alto y esta se puede recuperar rápidamente (Sánchez-Jabba, 2011).

Entre las externalidades negativas del mototaxismo, además de las ambientales por el aumento en la emisión de gases, se encuentra la afectación económica a las estructuras de transporte publico organizadas y legalmente constituidas, así como a los diferentes sistemas integrados de transportes que funcionan en las principales ciudades del país. Las afectaciones en la movilidad, las alteraciones de orden público, los índices de accidentalidad y la inseguridad son aspectos que preocupan a los Gobiernos municipales y departamentales.

De acuerdo con el Instituto de Hidrología, Meteorología y Estudios Ambientales (Ideam) y el Programa delas Naciones Unidas para el Desarrollo (PNUD) (2016), desde 1990 hasta 2014, las emisiones de gas carbónico $\left(\mathrm{CO}_{2}\right)$ en Colombia aumentaron un $10 \%$; es el quinto país de América Latina que más emite gases y el 34 a nivel mundial entre 184 países que tienen registros. El transporte y la industria manufacturera aportan el $12 \%$ del total de emisiones. Por otro lado, acorde con Cuéllar, Buitrago-Tello y Belalcazar-Ceron (2016), las motocicletas son los vehículos a gasolina que más producen emisiones de $\mathrm{CO}_{2}$; en promedio emiten 167 gramos por kilómetro recorrido, los taxis y los automóviles particulares producen un $40 \% \mathrm{y}$ $44 \%$ menos de emisiones, respectivamente. Según reportes del Registro Único Nacional de Tránsito (RUNT), de los 13.637 .663 vehículos matriculados y autorizados para circular en Colombia, el $57 \%$ son motocicletas. Solo en marzo de 2019 se vendieron en

1 De acuerdo con el Boletín de la Educación Superior en Cifras del Ministerio de Educación de Colombia, de diciembre de 2016, la tasa de cobertura bruta en educación superior del país es del $49,42 \%$. 
Colombia 50.100 motocicletas, un 20,7\% más en relación con el mismo mes de 2018; durante el primer trimestre de 2019 se vendieron 148.394 motos (Asociación Nacional de Movilidad Sostenible, 2019). Si bien no hay estudios que evidencien que la actividad del mototaxismo explica el aumento en la emisión de gases, las motocicletas sí generan un mayor volumen de contaminación en comparación con otros vehículos, y se ha evidenciado en los últimos años una creciente circulación de motocicletas en Colombia.

El Decreto 2961 de 2006, por el cual se dictan medidas para controlar la prestación del servicio público de transporte en motocicletas, autorizó a los municipios y distritos tomar las medidas necesarias para restringir la circulación de acompañantes o parrilleros por zonas de su jurisdicción o en horarios especiales, de acuerdo con la necesidad. En este sentido, las entidades territoriales empezaron a reglamentar el transporte y la circulación de las motocicletas, con el propósito no solo de controlar el mototaxismo, sino también de mitigar los crecientes problemas de inseguridad y delincuencia que están experimentando estos territorios, y que en algunos casos se atribuyen al fomento del mototaxismo.

La convivencia y la seguridad ciudadana son unas de las principales preocupaciones del Gobierno nacional y de los Gobiernos departamentales y municipales. Son muchas las encuestas que intentan medir la percepción de los colombianos de inseguridad y confianza en las instituciones de seguridad y justicia ${ }^{2}$, y los resultados son preocupantes: las personas no se sienten seguras y las estadísticas así lo demuestran. Según cifras del censo delictivo de la Fiscalía, en 2016 se denunciaron 314.511 robos, más de 850 casos diarios en todo el país. Si esto se compara con 2015, se presentó un aumento del $11 \%$.

2 La Cámara de Comercio de Bogotá y diferentes cámaras del país aplican una encuesta semestral de victimización y percepción de inseguridad. Solo en 2004 el Departamento Administrativo Nacional de Estadística (DANE) aplicó la encuesta de victimización y percepción de inseguridad en Bogotá, Cali y Medellín, después se dejó de aplicar. En 2012 nuevamente el DANE desarrollo y aplicó la encuesta de convivencia y seguridad ciudadana; también está aplicando la encuesta de victimización.
En este trabajo se estudia de qué manera las políticas públicas, adoptadas por las principales ciudades de Colombia para controlar el mototaxismo, han impacto la tasa de hurto a personas calculada por el DANE a través de la encuesta de Convivencia y Seguridad Ciudadana. Desde el punto de vista metodológico, se realiza una evaluación de impacto de políticas públicas, aplicando el método de diferencias en diferencias; se cuenta con información socioeconómica de las principales ciudades, para el periodo 2012-2017. Teniendo en cuenta la estructura de datos, se estimó un modelo econométrico de panel de datos de efectos fijos y aleatorios, así como un modelo de regresión lineal múltiple agrupado.

\section{Contextualización}

El fenómeno del mototaxismo, como actividad económica informal, ha sido objeto de grandes discusiones a lo largo y ancho del territorio nacional, y ha generado un gran conflicto social, luego de que esta actividad terminara convirtiéndose en una alternativa económica rentable y en una solución a corto plazo de las necesidades económicas y laborales de la población. Esto ha aumentado las cifras de informalidad que, según el DANE en su informe de medición del empleo informal y seguridad social, en 2017 fueron del 47,2\%, en las 13 ciudades y áreas metropolitanas, y del 48,74\% en las 23 ciudades y áreas metropolitanas.

Es importante comentar que no todo trabajo es empleo. De acuerdo con la oit (2013b), “[...] el trabajo comprende todas las actividades realizadas por personas de cualquier sexo y edad con el fin de producir bienes o prestar servicios para el consumo de terceros o para uso final propio" (p. 2); este se define independientemente de su carácter legal, formal o informal. El empleo, por su parte, hace referencia al trabajo que realizan las personas ya sea de manera formal o informal. El empleo formal hace referencia a los trabajadores que tienen una relación laboral reconocida con derechos laborales preestablecidos; el empleo informal abarca a los trabajadores que reciben un pago por su trabajo, pero sin una relación laboral reconocida que pueda hacer cumplir sus derechos laborales 
(OIT, 2013b). Por lo tanto, la actividad del mototaxismo puede considerarse un trabajo que realiza un grupo de individuos con el fin de prestar un servicio; sin embargo, debe ser reconocido como un empleo informal, pues no existe una relación laboral reconocida legalmente, y tampoco pueden hacer cumplir sus derechos laborales.

Por otro lado, el uso de motocicletas como medio de transporte público ha venido ganando un terreno importante entre las preferencias de movilidad de la población, con lo cual ha ocupado importantes renglones de la actividad económica informal. Esto complica el normal desarrollo de las actividades económicas formales, siendo cada vez más insostenible para las distintas entidades territoriales en materia de control de movilidad, espacio público, seguridad ciudadana, formalidad, índices de accidentabilidad, además del recaudo de impuestos por la prestación de servicios públicos de transporte debidamente formalizados. Este último es un tema crucial para toda administración pública, es decir, el costo de oportunidad en materia de recaudo en la administración crece año tras año, y los medios de transporte formal se ven afectados por una competencia que, según ellos, es desleal por no encontrarse en igualdad de condiciones frente a reglamentaciones e imposiciones de orden legal. Además, el mototaxismo es una amenaza constante para los distintos proyectos de sistemas masivos de transporte. Así, el mototaxismo es considerado como una competencia informal y desleal para el transporte público legal, y en algunas ciudades esta actividad es usada por ciertas personas como una fachada para cometer hurtos o actos ilegales.

El transporte en motocicleta es una actividad altamente riesgosa. Según las cifras de la Agencia Nacional de Seguridad Vial hasta 2018, se encuentran matriculas en el RUNT de Colombia cerca de 7.700.000 motocicletas, frente a 1.200.000 motos existentes en 2000. En el mismo periodo, se registraron en Colombia más de 198.000 accidentes de tránsito terrestre, que dejaron 6479 muertes y 38.070 personas lesionadas. El $49,4 \%$ de las víctimas fatales y el $56,2 \%$ de las personas lesionadas correspondieron a accidentes sufridos en motocicletas. Adicional a esto, el transporte en motocicleta es una de las actividades más contaminantes y ruidosas que se puedan concebir.

De acuerdo con la Dirección de Seguridad Ciudadana de la Policía Nacional, el hurto a personas es la modalidad de hurto más representativa en el país y la que más ha crecido durante los últimos años. En 2013, 1.725.000 de personas mayores de 15 años manifestaron haber sido víctimas de hurto en las 28 principales ciudades de Colombia, mientras que en 2017 esta cifra llegó a 1.849.000³.

La seguridad ciudadana es la principal preocupación de los colombianos, y esta preocupación crece en ciudades más grandes. De acuerdo con una encuesta de percepción de Invamer-Gallup realizada en 2015, el $63 \%$ de los barranquilleros, el $45,5 \%$ de los caleños, el $45,5 \%$ de los paisas, el $33,3 \%$ de los bumangueses y el 53,1\% de los bogotanos consideran que la inseguridad es el principal problema de su ciudad. Según Glaeser y Bruce (1999):

[...] el rendimiento económico de un delito medio es de aproximadamente un $20 \%$ más en las áreas metropolitanas que fuera de ellas. Cuando se duplica la población de una ciudad, las probabilidades de ser detenido por un delito cualquiera disminuyen en un $8 \%$, aproximadamente (p. 93).

La seguridad ciudadana se ha convertido en un tema crucial dentro de los planes de desarrollo de las diferentes entidades territoriales, de ahí la necesidad de tomar medidas que permitan disminuir los índices de inseguridad urbana. Una de estas medidas ha sido expedir decretos o acuerdos que regulen la circulación de motocicletas con parrilleros en las zonas más concurridas de las ciudades.

Es importante, entonces, establecer qué tan eficientes han sido estas medidas de política pública para combatir los altos índices de inseguridad, en especial el hurto a personas. En muchas regiones del país, se están imponiendo medidas que en la década de los 80 y 90 se adoptaron como estrategia para controlar los asesinatos ordenados por la mafia, la prohibición total del parrillero,

3 Cifras obtenidas de la Encuesta de Convivencia y Seguridad Ciudadana del DANE entre 2013 y 2017. 
principalmente en motos de alto cilindraje, es un ejemplo de ello.

De acuerdo con la firma Econometría Consultores, que desarrolló el estudio Caracterización de la prestación del servicio de transporte en motocicleta (mototaxismo) en Colombia en 2013, el mototaxismo surge como un servicio de transporte que atiende una necesidad de la comunidad por llegar a lugares donde ningún medio de transporte iba. A la vez, sirvió como alternativa de empleo. Hoy en día, el mototaxismo compite con los medio actuales de transporte público y los conductores asumen esta actividad como una opción real de empleo.

Por lo anterior, es importante estudiar la relación existente entre el mototaxismo y la criminalidad, medida a través del hurto a personas, y de qué manera las medidas de política implementadas por algunos Gobiernos locales han contribuido o no a reducir estos índices de hurto. La seguridad ciudadana es quizá uno de las dificultades que más aqueja el país, y el mototaxismo es uno de los principales problemas económicos, sociales y laborales del siglo XxI.

\section{Revisión teórica}

Según Sánchez-Jabba (2011), la principal razón que dio origen al mototaxismo fue el desempleo, siendo la tasa de paro de Colombia la segunda más alta en la región latinoamericana, 9,4\% en 2017. Debido a la carencia de oportunidades de empleo formal, algunas personas de escasos recursos deciden recurrir a su motocicleta para transportar personas y generar ingresos para el sostenimiento de sus familias. Otros individuos que no poseen una motocicleta trabajan como conductores de motocicletas de organizaciones informales.

En Colombia no existe evidencia empírica acerca del impacto que han tenido las políticas para controlar el mototaxismo sobre los índices de delincuencia, especialmente el hurto a personas, lo que hace que el presente trabajo sea pionero en esta temática. No obstante, existen otros estudios como el de Sánchez-Jabba (2011), quien estudio el impacto social y económico que generó el mototaxismo en Sincelejo, a través de un modelo probit bivariado. El autor encontró que el $88 \%$ de los mototaxistas tienen un nivel de educación máximo hasta secundaria, y solo el $5 \%$ tiene título universitario; por lo tanto, a mayor nivel de escolaridad, menor es la probabilidad de dedicarse al mototaxismo. La tenencia de vivienda también se correlaciona de forma negativa con la dedicación al mototaxismo.

Por otro lado, el Sisben representa un incentivo importante para dedicarse al mototaxismo, puesto que la mayoría tiene acceso a los programas sociales del Gobierno que incluyen régimen de cobertura subsidiada en salud. Otro factor que influye sobre la elección del mototaxismo es la marca de la moto; con base en los resultados, se puede establecer que si la moto que se conduce es de bajo costo, la probabilidad de ser mototaxista se incrementa. Este resultado se produce como respuesta a la estructura de costos, ya que el uso de este tipo de motos implica costos de operación e inversión bajos en comparación con otro tipo de marcas.

Gómez Morris y León Álvarez (2015) determinaron los principales efectos económicos de la incidencia delictiva en Cartagena durante el periodo 2004-2012. A través de la prueba de Granger realizaron un análisis causal teniendo en cuenta variables como la inversión extranjera directa e inversión neta de capital, tasa de ocupación hotelera e ingresos operacionales turísticos, inflación, ingresos laborales y gasto presupuestal en seguridad. Dentro de los principales resultados se encuentra que existe causalidad en la variable incidencia delictiva hacia la tasa de ocupación hotelera; además, se corrobora que existe causalidad entre la variable incidencia delictiva hacia los ingresos operacionales turísticos y en la variable incidencia delictiva hacia el gasto presupuestal en seguridad. Los autores encuentran que no existe causalidad de la incidencia delictiva hacia los ingresos laborales; asimismo, en el presente estudio se determina que la inversión extranjera directa (IED) no es afectada por la actividad delictiva de la ciudad, lo cual se explica gracias a que esta es una variable influida por factores principalmente económicos y no sociales, como es el carácter de la incidencia delictiva.

Por otra parte, Espinosa Gutiérrez et al. (2008) determinaron el impacto socioeconómico del 
fenómeno del mototaxismo en Montería. A través de entrevistas semiestructuradas, dirigidas a comerciantes, dueños de empresas y personas del común, los autores encontraron que los comerciantes que tienen algún vínculo con el mototaxismo se han visto beneficiados por este medio de transporte, ya que sus ventas dependen directamente del crecimiento de este fenómeno. Las empresas de transporte legal manifiestan que no tienen ningún alivio tributario que les permita seguir operando a pesar del efecto nocivo que genera el mototaxismo en su ocupación, pues al ser una actividad ilegal no tiene que pagar impuestos; esto los deja en una posición más favorable que las empresas constituidas de forma legal. Por último, los ciudadanos del común manifiestan su preocupación por la falta de autoridad por parte del Gobierno en el manejo de este fenómeno, que cada día crece y agrava los problemas económicos y sociales de las personas que viven de esta actividad.

Caro Aguirre y Cotinchara Soto (2018) realizaron una caracterización del perfil socioeconómico del gremio de los mototaxistas del municipio de San Martín de los Llanos. De acuerdo con los autores, las pocas oportunidades de empleo, las altas tasas de desempleo y la falta de atención de las instituciones estatales a esta actividad son aspectos que fomentan el desarrollo del mototaxismo. De la población dedicada al mototaxismo, el $34 \%$ tiene un nivel de educación de secundaria y el $53 \%$ presenta educación media.

García y González (2004) encontraron que en Cartagena el $72,04 \%$ de los mototaxistas pertenece a los estratos 1 y 2 ; el $68,8 \%$ tiene entre 17 y 34 años de edad, y el 76,35\% presenta un nivel de educación secundaria (completa o incompleta). Mónica Calderón, Gabriel Sevillano, Diana Segura y Yuli Rojas (PNUD, 2014) realizaron el estudio de los perfiles ocupacionales para el Cesar, con el cual encontraron que la edad de un poco más del $60 \%$ de la población dedicada al mototaxismo oscila entre los 18 y 29 años; asimismo, el $85 \%$ de la población pertenece a los estratos 1 y 2 . Respecto a la educación, el 59,8 \% de los mototaxistas tiene un nivel de educación secundaria (completa o incompleta), el 19,8\% tiene titulación como técnico y tecnólogo, y el 4,4\% es profesional. En general, las personas jóvenes con bajos niveles de educación y en los estratos socioeconómicos más bajos son las que en su mayoría desarrollan esta actividad.

\section{Implementación de políticas del mototaxismo}

El creciente uso de la motocicleta como medio de transporte no solo está relacionado con el aumento de la accidentalidad en las vías del país, sino que además se viene generando un aumento de la delincuencia o inseguridad ciudadana, aprovechando las ventajas que las motos ofrecen en materia de movilidad; esto es lo que suponen los Gobiernos locales de algunos municipios, en especial de las capitales con mayor densidad poblacional de Colombia. Por esta razón, las administraciones municipales y departamentales han implementado políticas para restringir esta actividad, considerada ilegal. A continuación, se describen las decisiones de política pública de las principales ciudades capitales del país.

\section{Pereira (Risaralda)}

Pico y placa $a^{4}$ para motos. Esta medida se aplica en toda la ciudad, según el primer número de la placa: lunes: 0-1; martes: 2-3; miércoles: 4-5; mueves, 6-7 y viernes: 8-9. Horarios: de 7:30 a. m. a 10:00 a. m., de 11:00 a.m. a 3:30 p.m. y de 5:00 p.m. a 7:30 p.m.

Están prohibidos parrilleros o pasajeros hombres mayores de 14 años, según el Decreto 485 del 27 junio 2008, modificado por el Decreto 418 del 9 de junio 2009.

\section{Villavicencio (Meta)}

El Decreto 228 de 2014 prohibió la circulación de motocicletas con acompañante hombre, con edades entre los 12 y los 65 años, las 24 horas del día.

4 El "pico y placa" es una medida de restricción vehicular implementada por los Gobiernos locales para controlar la movilidad de automóviles y motocicletas. La palabra "pico" se debe a la idea inicial era aplicarla solo en horas de mayor congestión vehicular (horas pico); sin embargo, en muchas regiones está medida se aplica en un número mayor de horas; y la palabra "placa" es porque la restricción de circulación vehicular se define dependiendo de los número (generalmente los últimos) de placa del automóvil. 


\section{Cúcuta (Norte de Santander)}

No se permite el tránsito de motocicletas de lunes a domingo y festivos en la franja de 11:00 p.m. a 5:00 a. m. Está restringida la circulación con acompañante hombre en cualquier horario y mujeres en estado de embarazo (Decreto 082 de 2010).

\section{Pasto (Nariño)}

En Pasto se implementa el pico y placa para motos de dos tiempos desde las 7:30 a. m. hasta 7:30 p. m., de lunes a viernes según el último digito de la placa (rota por día). Mediante el Decreto 0255 del 11 de marzo 2013, se restringe el tránsito de motocicletas con acompañante durante las 24 horas del día en la zona urbana. Esta norma no cobija a motocicletas que sean conducidas por sus propietarios, quienes podrán inscribir a seis personas siempre y cuando sean familiares; esto aplica solo para las motocicletas registradas en la ciudad.

\section{Popayán (Cauca)}

El Decreto 030 de 2010 prohibió la circulación de motocicletas con acompañantes en el centro de la ciudad; además, en la ciudad se aplica el pico y placa.

\section{Bucaramanga (Santander)}

En mayo de 2016, el Juzgado 15 Administrativo del Circuito Judicial de Bucaramanga dictaminó que los alcaldes del área metropolitana deben adoptar medidas prontas y eficaces para frenar el mototaxismo. Por lo anterior, mediante el Decreto 0174 del 1 de diciembre de 2016, Bucaramanga adoptó el plan de acción permanente para el control de la práctica informal del mototaxismo. En esta medida, en dicha ciudad se aplica pico y placa para motos.

\section{Cartagena (Bolívar)}

Se aplica pico y placa a motocicletas así: los días pares solo circulan motocicletas cuya placa termine en números pares y los días impares, solo aquellas con placa impar. Además, el Decreto 1329 de 2016, restringe la circulación de motocicletas de cualquier modalidad y cilindraje en todo el territorio del Distrito Turístico y Cultural de Cartagena, entre las 5:00 a. m. y las 11:00 p. m.

\section{Santa Marta (Magdalena)}

El Decreto 096 del 2 de marzo de 2010 estableció el pico y placa teniendo en cuenta el último dígito de la placa, así: lunes 1, 2, 3, 4; martes 5, 6, 7, 8; miércoles $9,0,1,2$ ), jueves $3,4,5$, 6 ; viernes $7,8,9,0$. Está prohibida la circulación con acompañante hombre todos los días de la semana en el centro histórico: desde la carrera 1 hasta la carrera 8 (Avenida del Ferrocarril) y desde la calle 10 hasta la 29, de 6:00 p. m. hasta las 12:00 m. los viernes, sábados $\mathrm{y}$ domingos.

\section{Valledupar (Cesar)}

El Decreto 308 de 2014 establece que solo el propietario de la motocicleta puede conducirla y que durante días hábiles se prohíbe el ingreso de estos vehículos a ciertas zonas del centro de la ciudad. Además, prohíbe el transporte de parrilleros hombres mayores de 14 años.

\section{Neiva (Huila)}

El Decreto 080 de 2015, establece que solo el propietario o titular del vehículo podrá circular con parrillero. Asimismo, está prohibida la circulación de motocicletas o similares con acompañante en el microcentro. La restricción se aplica de lunes a viernes entre las 7:00 a. m. y las 7:00 p. m.

\section{Cali (Valle del Cauca)}

El Decreto 0412 de junio de 2007 establece, prohíbe, entre otras, la circulación de todo tipo de parrillero hombre mayor de 14 años de edad; de igual manera, la circulación de motos entre las 0:00 horas y las 5:00 a. $\mathrm{m}$.

\section{Barranquilla (Atlántico)}

El Decreto 0838 de 2014 prohibió a los conductores de motocicletas el transporte de parrilleros, con excepción de la esposa/o, hijos (adoptivos o consanguíneos) o "hasta cinco personas adicionales", debidamente registradas en la Secretaría de Movilidad. 


\section{Medellín (Antioquia)}

En agosto de 2015 en Medellín, el Tribunal Administrativo derogó el Decreto 1369 que restringía la circulación de motocicletas con parrillero, argumentando que había una afectación innecesaria para miles de ciudadanos de bien. Por lo anterior, desde 2015 en Medellín las motocicletas pueden circular libremente con parrillero.

\section{Armenia (Quindío)}

En el departamento de Quindío, el Gobierno departamental en 2012 derogó el Decreto 0258 que prohibía el transporte de parrillero hombre en motos y permitía la circulación de estas solo hasta las 11:00 p. m. Por lo tanto, en todos los municipios del Quindío, incluyendo Armenia, se permite la movilización de parrillero sin importar el sexo, con libre tránsito hasta las 12:00 m. de la noche.

Por último, en Manizales, Ibagué y Sincelejo hasta 2017 no existía regulación por parte de las autoridades locales para controlar el fenómeno del mototaxismo. Recientemente, en Ibaguése prohibió la circulación de motocicletas con cualquier clase de parrillero en toda la ciudad desde las 11:00 p. m. hasta las 6:00 a. m., pero solo durante el Festival Folclórico de Ibagué de 2019.

\section{Metodología}

La evaluación del impacto de políticas permite probar si la política o estrategia implementada se asocia a los resultados obtenidos. Respecto a las políticas públicas, como en este caso, el seguimiento y la evaluación brindan elementos esenciales para la administración gubernamental, pues retroalimentan constantemente la toma de decisiones.

En la literatura académica existen diversas metodologías para evaluar el impacto de políticas; una de las más comunes es el método de diferencias en diferencias. De acuerdo con Wooldridge (2015), diferencias en diferencias es una técnica cuasiexperimental, que cuenta con un grupo control, que no se ve afectado por el cambio de política, y un grupo tratamiento, que sí se ve afectado por este cambio. Para calcular las diferencias sistemáticas entre estos dos grupos se necesitan datos para dos momentos: un periodo antes de la aplicación de la política y otro después de la aplicación. Por lo tanto, generalmente la muestra se descompone en dos grupos: el grupo control antes y después de la política, y el grupo tratamiento antes y después de la política. Sea dT una variable binaria que toma el valor de 1 para el grupo tratamiento y 0 para el grupo control; $\mathrm{d} 2$, otra variable binaria que toma el valor de 1 después del cambio de la política y 0 antes del cambio, y Y, la variable resultante que nos interesa, la ecuación de interés es:

$Y=\beta_{0}+\delta_{0} d 2+\beta_{1} d T+\delta_{1} d 2 * d T+$ otros factores

$\delta_{1}$ mide el efecto de la política, y también es llamado estimador de diferencias en diferencias, ya que calcula el cambio en los promedios respecto al tiempo para cada uno de los grupos de tratamiento y control, y después determina la diferencia entre estos dos cambios.

En este estudio, del grupo control hace parte un conjunto de municipios que no han implementado políticas públicas para controlar la circulación de motocicletas; el grupo tratamiento lo conforman los municipios que sí han implementado políticas públicas. Así, se definió como año base el 2014; por lo tanto, la variable d2 tomará el valor de 1 después de 2014, y el valor de 0 antes de 2014. De esta manera, se dispone de información para cuatro grupos: el grupo de municipios que no implementó ninguna política (control), tanto antes como después de 2014, y el grupo de municipios que sí implementó alguna política (tratamiento), antes y después.

Es importante mencionar que los municipios del grupo tratamiento y control son los mismos antes y después de 2014; así, pues, se está haciendo seguimiento a los mismos municipios a través del tiempo. Por lo anterior, la estructura de datos más adecuada para estimar el modelo de diferencias en diferencias corresponde a un conjunto de datos de panel, que de acuerdo con Wooldridge (2015) consiste en una serie de tiempo por cada unidad de una base de datos de corte transversal. Este tipo de estructura de datos es muy útil para el análisis de políticas. Uno de los principales objetivos de aplicar los métodos de datos de panel es capturar la heterogeneidad inobservable, ya sea entre las unidades analizadas o en el tiempo, dado que 
la heterogeneidad no se puede tratar con datos de corte transversal ni con datos de series de tiempo.

De esta forma, la ecuación planteada corresponde a:

tasahurtopersonas $_{i t}=\beta_{0}+\delta_{0} y 14_{t}+\beta_{1} \times$ politica $_{i t}$ $+\delta_{1}\left(y 14_{t} \times\right.$ politica $\left._{i t}\right)+$ otros factores $+a_{i}+\mu_{i t}$

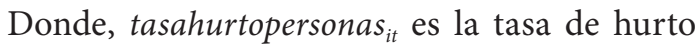
a personas de la ciudad i durante el año t, $y 14_{t}$ es una variable binaria que toma el valor de 1 después de 2014 y 0 antes de 2014; politica $_{i t}$ es otra variable binaria que tomará el valor de uno si la ciudad i en el año t implementó alguna política para controlar el mototaxismo. $\left(y 14_{t} \times\right.$ politica $\left._{i t}\right)$ es nuestra variable de mayor interés, pues indica la efectividad de la política, ya que compara a las ciudades que implementaron alguna política después de 2014 con aquellas que no lo hicieron. Finalmente, $a_{i}$ es el efecto inobservable fijo de cada ciudad, que se elimina con la aplicación de los métodos de panel de datos.

En este trabajo se aplicaron dos especificaciones del modelo de panel de datos: 1) efectos fijos (MEF), el cual utiliza un mecanismo de transformación, que consiste en expresar cada variable como una desviación de su valor medio para eliminar la heterogeneidad inobservable antes de la estimación y 2) efectos aleatorios (MEA), que como mecanismos de transformación utiliza un parámetro $\lambda$, como proporción de los valores medios temporales, el cual está entre 0 y 1 , y depende del tiempo y de las varianzas $a_{i}+\mu_{i t}$. Además, se estimó un modelo de regresión lineal múltiple agrupado, es decir, sin tener en cuenta el efecto temporal y la distribución por ciudades, con fines comparativos.

Sin embargo, es importante identificar cuál de los modelos estimados es el que ofrece mejores resultados. En este sentido, se consideró la prueba de Breusch-Pagan y la prueba de Hausman, que permiten identificar la presencia de efectos constantes en el término error de un modelo estimado; de este modo, brindan un referente para elegir entre un modelo de efectos fijos y uno de efectos aleatorios. Hausman permite identificar la existencia de diferencias sistemáticas entre las estimaciones por el método de efectos fijos y aleatorios.

Dado que el objetivo de este trabajo es determinar el impacto sobre la tasa de hurto a personas y de las diferentes políticas que se han implementado en las principales ciudades de Colombia para controlar el mototaxismo, el modelo estimado tiene como variable dependiente la tasa de hurto a personas, que establece el porcentaje de población de 15 años y más que ha sufrido cualquiera de las modalidades de hurto a personas, por ejemplo, raponazo, atraco, descuido, entre otros. Los datos provienen de la Encuesta de Convivencia y Seguridad Ciudadana del DANE.

Las variables que fueron incluidas en este trabajo se construyeron a partir de las estadísticas publicadas por el DANE entre 2012 y 2017, así como información suministrada por la Policía Nacional. Dentro de estas variables se encuentran la tasa de desempleo, la tasa de inflación, el índice de pobreza, porcentaje de población en estrato bajo (estrato 1 y 2) y el producto interno bruto (PIB, por departamento a precios constantes del año base 2015), todas medidas en porcentajes; asimismo, la escolaridad (promedio de años de educación de la población mayor de 15 años), el índice de Gini y el número de cuadrantes y de CAI de Policía por ciudad. Los resultados se estimaron utilizando el software Stata 15.

\section{Resultados}

Con base en la metodología planteada, una parte importante de los resultados es determinar las características de la muestra. Se cuenta con información para seis periodos (2012-2017) de 17 ciudades capitales de Colombia, por lo que se dispone de una muestra total de 102 observaciones para los seis periodos. Del total de ciudades, ocho ya habían aplicado alguna política para controlar el mototaxismo antes de 2014, año que se tomó como corte para evaluar la efectividad de dichas políticas. Después del 2014, cuatro ciudades más implementaron alguna política, para un total de 12 ciudades. Con respecto a las ciudades que no implementaron ninguna política, antes de 2014 eran nueves las que no se habían pronunciado al respecto, pero después de 2014 el número de ciudades se redujo a cinco.

El anexo 1 muestra la media y la desviación estándar durante el periodo 2012-2017 para cada una de las ciudades estudiadas. Pasto registra la 
Tabla 1. Ciudades que aplicaron políticas para controlar el mototaxismo

\begin{tabular}{|c|c|c|}
\hline & Antes de 2014 & Después de 2014 \\
\hline Sin Política & $\begin{array}{l}\text { Bucaramanga, Barranquilla, Cartagena, Cesar, } \\
\text { Ibagué, Manizales, Neiva, Sincelejo y Villavicencio }\end{array}$ & Armenia, Ibagué, Manizales, Medellín y Sincelejo \\
\hline Con Política & $\begin{array}{l}\text { Armenia, Cúcuta, Cali, Medellín, Pasto, Pereira, } \\
\text { Popayán y Santa Marta }\end{array}$ & $\begin{array}{l}\text { Bucaramanga, Barranquilla, Cúcuta, Cali, Cartagena, Cesar, Neiva, } \\
\text { Pasto, Pereira, Popayán, Santa Marta y Villavicencio }\end{array}$ \\
\hline
\end{tabular}

Fuente: elaboración propia.

tasa más alta de hurto a personas: el 20,7\% de la población mayor de 15 años ha sido víctima de cualquiera de las modalidades de hurto a personas. Cartagena presenta la tasa más baja: 5,45\% durante el periodo 2012-2017. La tasa de desempleo promedio más alta se observa en Armenia $(14,5 \%)$ y la más baja se reporta en Bucaramanga $(7,9 \%)$. Manizales registra la tasa de inflación más alta durante 2012-2017 (4,6\%), mientras que en Cúcuta se reporta la tasa más baja (3,25\%). El departamento de Antioquía presenta el PIB más alto, con $\$ 117.325 .000$, y Sucre reporta el más bajo, con $\$ 6217$ miles de millones). En cuanto a los indicadores sociales, Cali, Manizales, Pasto y Popayán registran el mismo coeficiente Gini $(0,49)$, y son las ciudades con mayor desigualdad. Bucaramanga registra el nivel de desigualdad más bajo $(0,42)$; Santa Marta registra las tasas más altas de pobreza: el $33,8 \%$ de la población es pobre, mientras que en Bucaramanga el 10,1\%.

El $85,89 \%$ de la población de Neiva pertenece a los estratos 1 y 2 , lo cual la hace la ciudad con el mayor registro en estratos bajos, y Manizales tiene la menor proporción es estos niveles de estratos $(41,98 \%)$. Cali registra el índice de escolaridad más bajo: el 8,7\% de la población de 15 años y más no sabe leer y escribir, mientras que Cartagena reporta el índice de escolaridad promedio más alto, 10,18\%. Con respecto a la información de la Policía, Manizales registra menor número de cuadrantes de policía por cada 1000 habitantes $(0,59)$; por su parte, Pereira reporta el promedio más alto: 2,16 cuadrantes de Policía por cada 1000 habitantes. En Bucaramanga existen 7,1 CAI de policía por cada 1000 habitantes y en Cali, solo 1,7.

La metodología más simple para la estimación de un modelo de panel de datos supone omitir las dimensiones del tiempo y el espacio, y estimar un modelo de regresión por mínimos cuadrados ordinarios (MCO) agrupados (Pool OLs). Los resultados de las estimaciones se muestran en el primer recuadro de la tabla 2. Sin embargo, el modelo por MCO, parte del supuesto de que el intercepto de la regresión es igual para todos los individuos, en este caso, ciudades. Sin embargo, es importante que se pueda controlar el carácter individual de cada ciudad, para lo cual se consideró el modelo de efectos aleatorios, que supone que cada ciudad tiene un intercepto diferente. Las estimaciones del modelo de efectos aleatorios se muestran en el segundo recuadro de la tabla 2 .

Para determinar si es necesario estimar el modelo de mínimos cuadrados ordinarios agrupados o efectos aleatorios, se realizó la prueba del multiplicador de Lagrange para efectos aleatorios de Breusch y Pagan. La hipótesis nula de esta prueba sostiene que la varianza del error es cero $\left(\sigma_{\mu}{ }^{2}=0\right)$. Los resultados permiten rechazar la hipótesis nula, por lo que es preferible estimar el modelo por efectos aleatorios (la estimación de la prueba se muestra en el anexo 2).

El carácter individual de cada ciudad también se puede controlar a través de un modelo de efectos fijos, el cual supone que las diferencias entre ciudades son fijas o constantes, y no aleatorias. Las estimaciones del modelo de efectos fijos se muestran en el tercer recuadro de la tabla 2. La prueba $\mathrm{F}$ de significancia de los efectos fijos ${ }^{5}$ indica que es preferible usar el método de efectos fijos que el método de mínimos cuadrados ordinarios agrupado.

5 Esta prueba $\mathrm{F}$, que es restringida porque asume un mismo intercepto para todos las ciudades, compara el modelo Pool OLS con el modelo de efectos fijos. Los resultados de la prueba son: $F(16,73)=9,53$. Prob $>F=0,000$. 
Tabla 2. Estimación con datos de panel

\begin{tabular}{|c|c|c|c|c|c|c|}
\hline \multirow{2}{*}{$\begin{array}{l}\text { Variable dependiente } \\
\text { Log(tasa de criminalidad) }\end{array}$} & \multicolumn{2}{|c|}{ Pool OLS } & \multicolumn{2}{|c|}{ Random-effects } & \multicolumn{2}{|c|}{ Fixed-effects } \\
\hline & Coef & SE & Coef & SE & Coef & SE \\
\hline Año & $-0,3040^{* *}$ & 0,1344 & $-0,7743$ & 0,1184 & 0,1039 & 0,1222 \\
\hline Política & $0,2699 * *$ & 0,1282 & $0,2164^{* *}$ & 0,0967 & $0,2614^{* * *}$ & 0,9364 \\
\hline Política*año & $-0,0282$ & 0,1455 & $-0,1589$ & 0,1055 & $-0,2341^{* *}$ & 0,1003 \\
\hline Log(desempleo) & $0,7717^{* * *}$ & 0,2003 & $0,4004^{*}$ & 0,2069 & $0,36789 *$ & 0,2185 \\
\hline Log(inflación) & $0,1193^{*}$ & 0,0624 & $0,1064^{* *}$ & 0,0445 & 0,0685 & 0,0424 \\
\hline Log(PIB Dptal constante) & $-0,1989 * * *$ & 0,0610 & $-0,0465$ & 0,1127 & $1,2948^{*}$ & 0,7500 \\
\hline Log(índice pobreza) & $-0,6359 * * *$ & 0,1675 & 0,0807 & 0,2344 & $0,6084^{* *}$ & 0,2863 \\
\hline $\log ($ Gini) & $6,3336^{* * *}$ & 1,6967 & $4,3278^{*}$ & 1,7364 & $3,8906^{* *}$ & 0,8670 \\
\hline Log(población en estrato bajo) & $0,6296 * * *$ & 0,2047 & $-0,2025$ & 0,3669 & $-2,2476^{* * *}$ & 0,8019 \\
\hline Escolaridad (años) & $0,3467^{* * *}$ & 0,1024 & 0,0277 & 0,1393 & $-0,4779^{* *}$ & 0,2081 \\
\hline Cuadrantes Policía/1000 hab. & $-0,00228$ & 0,6726 & 0,00538 & 0,6394 & $0,01252^{*}$ & 0,0657 \\
\hline CAI Policía/1000 hab. & $-0,02078$ & 0,2552 & $-0,02629$ & 0,2512 & $-0,05936^{* *}$ & 0,2744 \\
\hline Constante & $-4,6088^{* * *}$ & 1,6181 & $-0,1429$ & 2,4952 & $-1,4460$ & 6,3608 \\
\hline \multirow{2}{*}{ F / Wald test } & $\mathrm{F}$ & 0,000 & chi20 & 36,49 & $\mathrm{~F}$ & 4,17 \\
\hline & Prob. & 5,80 & Prob. & 0,0003 & Prob. & 0,0001 \\
\hline Within & R-squared & 0,4387 & \multicolumn{2}{|c|}{0,3154} & \multicolumn{2}{|c|}{0,4068} \\
\hline Overall & & & \multicolumn{2}{|c|}{0,2197} & \multicolumn{2}{|c|}{0,0001} \\
\hline Between & & & \multicolumn{2}{|c|}{0,1851} & \multicolumn{2}{|c|}{0,0016} \\
\hline Observations & \multicolumn{2}{|c|}{102} & \multicolumn{2}{|c|}{102} & \multicolumn{2}{|c|}{102} \\
\hline Grupo de individuos & & & \multicolumn{2}{|c|}{17} & \multicolumn{2}{|c|}{17} \\
\hline
\end{tabular}

Nota: $* * *, * *, *$ denotes $1 \%, 5 \%$ y $10 \%$ significance level, respectively.

Fuente: elaboración propia.

Tanto la prueba de efectos aleatorios de Breusch y Pagan, como la prueba F de significancia de los efectos fijos indican que el modelo de efectos aleatorios y de efectos fijos se prefieren más que el modelo agrupado. Para establecer cuál de los dos modelos - efectos fijos o aleatorios- es el más adecuado, es importante identificar la posible correlación entre el error individual y las variables explicativas $X$. En el modelo de efectos aleatorios se supone que esta correlación es cero. El test de Hausman define como hipótesis nula la no correlación entre $\mu_{i} \mathrm{y} X$, y establece que los estimadores de efectos fijos y aleatorios no difieren sustancialmente. En el anexo 3 se muestran los resultados de la estimación de la prueba de Hausman, y se concluye que se rechaza la hipótesis nula, por lo que sí existe diferencia entre los dos estimadores; por lo tanto, conviene estimar el modelo a través del método de efectos fijos.

Los tres modelos estimados no presentan diferencias sustanciales. El efecto de mayor interés, el

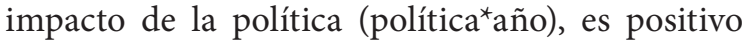

en los tres modelos, pero solo significativo en el modelo de efectos fijos. El desempleo, la inflación y el coeficiente de Gini impactan de manera positiva la tasa de hurto en los tres modelos, y el impacto del número de CAI por habitante es negativo. Las estimaciones difieren en variables como escolaridad, población en estratos bajos y el índice de pobreza.

Uno de los problemas comunes de los datos de panel es la violación del supuesto de autocorrelación o correlación serial, que ocurre cuando los errores de diferentes unidades están correlacionados o cuando los errores dentro de cada unidad se correlacionan en el tiempo. Para determinar si el modelo estimado presenta problemas de autocorrelación, se tuvo en cuenta la prueba de Autocorrelación para Panel de Datos de Wooldridge. Los resultados de la prueba (anexo 4) indican que no existe suficiente evidencia empírica para rechazar la hipótesis nula de no autocorrelación.; así, los modelos estimados por datos de panel no presentan problemas de autocorrelación. 
La dificultad de estimar un modelo de efectos fijos es que se puede presentar el problema de la heteroscedasticidad o autocorrelación entre las variables explicativas, de modo que se pueda afectar la inferencia estadística. Para determinar este problema, se consideró la prueba modificada de Wald para Heteroscedasticidad, que define como hipótesis nula la no existencia de heteroscedasticidad. En el anexo 5 se muestran los resultados de la estimación de la prueba, la cual indica que se rechaza la hipótesis nula de varianza constante y se concluye que existe heterocedasticidad. Para solucionar este problema se estimaron los tres modelos con errores estándar corregidos o robustos a la heteroscedasticidad, de manera que la inferencia sea correcta y se pueda confiar en la significancia estadística de las variables. Los resultados de la estimación por desviaciones típicas robustas se muestran en la tabla 3.

Después de determinar que el modelo de panel de efectos fijos con errores robustos es el más indicado para analizar el impacto de las políticas para controlar el mototaxismo en relación con el hurto a personas, se encontró que las variables significativas son el efecto de la política, el desempleo, el PIB departamental, el índice de pobreza, el coeficiente de Gini, la población en estratos bajos, la escolaridad, el número de cuadrantes y de cai de Policías por habitante.

Así, el efecto de mayor interés se evidencia en la variable política*año, la cual indica el efecto de dobles diferencias de las políticas implementadas por las alcaldías de las principales ciudades para controlar la circulación de motocicletas con parrillero. El coeficiente estimado indica que en los municipios donde se implementó la política después de 2014 la tasa de hurto a personas disminuyó en un $23,4 \%$, lo que muestra que la política fue efectiva en la diminución del hurto a personas. Este, sin duda, es el resultado más importante de este artículo.

Tabla 3. Estimación con datos de panel robustos a la heteroscedasticidad

\begin{tabular}{|c|c|c|c|c|c|c|}
\hline \multirow{2}{*}{$\begin{array}{l}\text { Variable dependiente } \\
\text { Log(tasa de criminalidad) }\end{array}$} & \multicolumn{2}{|l|}{ Pool OLS } & \multicolumn{2}{|c|}{ Random-effects } & \multicolumn{2}{|l|}{ Fixed-effects } \\
\hline & Coef & SE-Robt. & Coef & SE-Robt. & Coef & SE-Robt. \\
\hline Año & $-0,3040 * *$ & 0,1154 & $-0,774$ & 0,1066 & 0,1039 & 0,1047 \\
\hline Política & $0,2699 * *$ & 0,1144 & $0,2164^{* *}$ & 0,0984 & $0,2614^{* *}$ & 0,1154 \\
\hline Política*año & $-0,0282$ & 0,1261 & $-0,1589 * *$ & 0,0706 & $-0,234^{* * *}$ & 0,0621 \\
\hline Log(desempleo) & $0,7717^{* * *}$ & 0,1741 & $0,40045^{*}$ & 0,2425 & $0,367^{*}$ & 0,1973 \\
\hline Log(inflación) & $0,1193^{* *}$ & 0,0490 & $0,10641^{* *}$ & 0,0444 & 0,0685 & 0,0399 \\
\hline Log(PIB Dptal constante) & $-0,1982^{* * *}$ & 0,0678 & $-0,0465$ & 0,0975 & $1,294^{*}$ & 0,6395 \\
\hline Log(índice pobreza) & $-0,6359 * * *$ & 0,1955 & 0,0807 & 0,2507 & $0,6084^{* *}$ & 0,2659 \\
\hline $\log ($ Gini) & $6,3336^{* * *}$ & 1,6080 & $4,3278 * * *$ & 1,2680 & $3,8906^{* *}$ & 1,378 \\
\hline Log(población en estrato bajo) & $0,62965^{* * *}$ & 0,2080 & $-0,2025$ & 0,3356 & $-2,247^{* * *}$ & 0,6208 \\
\hline Escolaridad (años) & $0,34679 * * *$ & 0,7707 & 0,0277 & 0,1170 & $-0,4779 * *$ & 0,1956 \\
\hline Cuadrantes Policía/1000 hab. & $-0,00228$ & 0,571 & 0,005381 & 0,0459 & $0,01252^{*}$ & 0,0613 \\
\hline CAI Policía/1000 hab. & $-0,02078$ & 0,2377 & $-0,02629$ & 0,1691 & $-0,0593 * *$ & 0,2075 \\
\hline Constante & $-4,6088^{* * *}$ & 1,672 & $-0,1429$ & 2,341 & $-1,4460$ & 5,267 \\
\hline \multirow{2}{*}{ F/ Wald test } & $F$ & 9,10 & chi2 & 156,90 & $\mathrm{~F}$ & 33,42 \\
\hline & Prob. & 0,000 & Prob. & 0,000 & Prob. & 0,000 \\
\hline Within & R-squared & 0,4387 & \multicolumn{2}{|c|}{0,3154} & \multicolumn{2}{|c|}{0,4068} \\
\hline Overall & & & \multicolumn{2}{|c|}{0,2197} & \multicolumn{2}{|c|}{0,0001} \\
\hline Between & & & \multicolumn{2}{|c|}{0,1851} & \multicolumn{2}{|c|}{0,0016} \\
\hline Observations & \multicolumn{2}{|c|}{102} & \multicolumn{2}{|c|}{102} & \multicolumn{2}{|c|}{102} \\
\hline Grupo de individuos & & & \multicolumn{2}{|c|}{17} & \multicolumn{2}{|c|}{17} \\
\hline
\end{tabular}

Nota: $* * *, * *, *$ denotes $1 \%, 5 \%$, y $10 \%$ significance level, respectively.

Fuente: elaboración propia. 
Otros resultados muestran que la tasa de desempleo se relaciona con la tasa de hurto a personas, por un aumento de un punto porcentual en la tasa de desempleo. El hurto a personas crece en un $0,36 \%$, lo que mantiene constante el efecto de las demás variables. Algo similar ocurre con el índice de pobreza y el coeficiente de Gini: por un aumento del $1 \%$ en cada una de estas variables, el hurto a personas disminuye en $0,6 \%$ y $3,89 \%$, respectivamente. A mayores años de escolaridad promedio por ciudad, menor es la tasa de hurto. En términos generales, se observa que las condiciones socioeconómicas menos favorables se relacionan con mayores tasas de hurto a personas.

Por otro lado, a medida que el PIB departamental es mayor, la tasa de hurto a personas de la ciudad capital tiende también a ser mayor; un aumento del $1 \%$ en el PIB departamental a precios constantes acrecienta la tasa de hurto a personas en $1,29 \%$. Economías más grandes se asocian con mayor tasa de hurto a personas, pues en estas economías existe una población más grande con mejores condiciones económicas, que posiblemente es víctima de hurtos. Por otro lado, a medida que se incrementa la población en estratos 1 y 2 (aumenta en un punto porcentual), disminuye la tasa de hurto a personas (en 2,24\%); a mayor población en estratos bajos, mayor seguridad, pues es menor la cantidad de personas en estratos medio y alto que puedan ser víctimas de alguna de las modalidades de hurto a personas.

Un mayor número de CAI de Policía se relaciona con una menor tasa de hurto a personas; a medida que se aumenta un CAI por cada mil habitantes, la tasa de hurto a personas se disminuye 5,9\%, lo que permite constancia en el efecto de las demás variables. Sin embargo, el efecto del número de cuadrantes de policía es un poco contradictorio, ya que por un aumento de un cuadrante de policía por cada mil habitantes, la tasa de hurto a personas aumenta $1,2 \%$. Esto indica que la política de cuadrantes de Policía no parece ser la más efectiva para reducir el hurto a personas; no obstante, es importante tener en cuenta que este efecto es pequeño y estadísticamente significativo, pero al $10 \%$.

Finalmente, en cuanto a la bondad de ajuste, los tres modelos estimados son globalmente significativos (prueba $\mathrm{F} \mathrm{y} \mathrm{chi}{ }^{2}$ ). Respecto al modelo de efectos fijos, toda la variación de las variables explicativas se origina dentro de cada grupo, en este caso, cada ciudad; de esta forma, la diversidad del conjunto de variables independientes dentro de cada ciudad a través del tiempo explica el 40,68\% de la variación de la tasa de hurto a personas.

\section{Conclusiones}

El mototaxismo es un fenómeno que viene incrementándose de manera considerable en todo el territorio colombiano. Ha surgido como una alternativa informal de trabajo, en especial para personas con limitadas probabilidades de ingresar al mercado laboral formal. Los impactos económicos del mototaxismo son importantes, pues la crisis de la mayoría de los sistemas integrados y estratégicos de transporte es atribuible al ejercicio del mototaxismo. Las diferentes empresas del transporte público, en especial urbano, ven en el mototaxismo su principal competencia.

En materia de criminalidad y seguridad ciudadana, la mayoría de sus modalidades viene disminuyendo; los homicidios en 2017 se redujeron un $10 \%$, y el secuestro y las extorsiones cayeron un $8 \%$ y $12 \%$, respectivamente; sin embargo, el hurto a personas es el delito que más impacta a los colombianos, ya que aumentó un $27,6 \%$ en 2017 , específicamente el hurto a celulares, que creció un $53 \%$.

En algunas ciudades se atribuye este problema principalmente a la libre circulación de motocicletas con parrilleros, más que nada en el centro de las ciudades, donde hay mayor concurrencia de personas. Esto llevó a que diferentes ciudades adoptaran acuerdos que regularan la circulación de motocicletas con parrillero.

Es importante mencionar que no se ha podido establecer cuál es el impacto real del mototaxismo sobre la seguridad, o en concreto el hurto a personas, ya que no se conoce con exactitud el número de individuos que se dedican a esta actividad. En este artículo se determinó cuál ha sido el impacto de las políticas adoptadas por las diferentes ciudades de Colombia para controlar el mototaxismo sobre el hurto a personas; pero esto no significa que se haya determinado el impacto del mototaxismo 
sobre el hurto a personas, o la relación entre el mototaxismo y el hurto a personas.

La principal conclusión de esta investigación es que las medidas adoptadas para controlar la circulación de motocicletas con parrillero en las diferentes ciudades de Colombia han tenido un efecto significativo sobre la reducción en la tasa de hurto a personas; es decir, estas políticas han sido efectivas para reducir el hurto en las ciudades. También se encontró que las condiciones socioeconómicas de las ciudades se relacionan directamente con el hurto a personas; a mayores tasas de desempleo, índice de pobreza y coeficiente Gini, mayores tasas de hurto a personas. En ciudades con economías departamentales grandes también se presenta mayor cantidad de hurtos.

El número de CaI de Policía por ciudad está vinculado de manera positiva con el hurto a personas, mientras que el número de cuadrantes de policía presentan un efecto negativo. Todo parece indicar que la política de cuadrantes de policía no contribuye a reducir el hurto a personas.

Por último, la propuesta es que en futuros trabajos se evalué la efectividad de las medidas de política, adoptadas por las entidades territoriales para controlar la circulación de motocicletas con parrillero a nivel individual por municipio, pues las medidas aplicadas en los diferentes municipios no son necesariamente las mismas. Es importante entender este análisis de esta manera, ya que cada territorio tiene sus propias particularidades.

\section{Referencias}

Alcaldía de Bucaramanga. (1 de diciembre de 2016). "Decreto 0174 ". Bucaramanga, Colombia.

Alcadía de Cesar. (2014). "Decreto 308. Cesar". Cesar, Colombia.

Alcaldía de Cúcuta. (2010). “Decreto 085”. Cúcuta, Colombia.

Alcaldía Distrital de Barranquilla. (2014). "Decreto 0838”. Barranquilla, Colombia.

Alcaldía Distrital de Cartagena. (2016). "Decreto 0424". Cartagena, Colombia.

Alcaldía de Medellín (2013). “Decreto 1369”. Medellín, Colombia.

Alcaldía Municipal de Villavicencio. (2014). "Decreto 228”. Villavicencio, Colombia.
Alcaldía de Neiva. (2015). “Decreto 080”. Neiva, Colombia. Alcaldía de Pasto. (11 de marzo de 2013). "Decreto 0255". Pasto, Colombia.

Alcaldía de Pereira. (9 de junio de 2009). "Decreto 418". Pereira, Colombia.

Alcaldía de Pereira. (27 de junio de 2008). “Decreto 485”. Pereira, Colombia.

Alcaldía de Popayán. (2019). “Decreto 030”. Popayán, Colombia.

Alcaldía de Santa Marta. (2 de marzo de 2016). "Decreto 096”. Santa Marta, Colombia.

Alcaldía de Santiago de Cali. (junio de 2007). "Decreto 0412". Santiago de Cali, Colombia.

Arboleda Lozano, D. D. y Cobo Cartagena, J. C. (2016). Caracterización de las condiciones del bienestar laboral y psicosocial en el trabajo informal de mototaxismo en el Municipio de Palmira. (Tesis de grado). Universidad del Valle, Palmira, Colombia. Recuperado de http://bibliotecadigital.univalle.edu.co/bitstream/10893/10113/1/CB0554312.pdf

Ardila, A. (2006). El mototaxismo: causas y posibles soluciones. Boletín informativo en línea. Recuperado de http://www.voltairenet.org/article143679.html

Asociación Nacional de Movilidad Sostenible. (2019). Informe motos marzo. Colombia 2019. Recuperado de http:// www.andemos.org/wp-content/uploads/2019/04/Informe-Motos-2019-03.pdf

Caro Aguirre, L. P. y Cotinchara Soto, L. J. (2018). Caracterización del perfil socioeconómico del gremio de los mototaxistas del municipio de San Martín de los Llanos. (Tesis de grado). Universidad de Los Llanos, Villavicencio, Colombia.

Conferencia Internacional de Estadísticas de Trabajo. (1993). Resolución sobre las estadisticas del empleo en el sector informal. Decimoquinta conferencia Internacional de Estadísticas del Trabajo .

Cuéllar, Y., Buitrago-Tello, R. y Belalcazar-Ceron, L. C. (2016). Life cycle emissions from a bus rapid transit system and comparison with other modes of passenger transportation. CT\&F-Ciencia, Tecnología y Futuro, 6(3), 123-134.

Departamento Administrativo Nacional de Estadísticas (DANE). (2012). Encuesta Nacional de Calidad de Vida. Recuperado de: https://www.dane.gov.co/files/ investigaciones/condiciones_vida/calidad_vida/Boletin_Prensa_ECV_2012.pdf

Departamento Administrativo Nacional de Estadísticas (DANE). (2015). Cuentas departamentales - Colombia. Producto Interno Bruto (PIB). Recuperado de https:// 
www.dane.gov.co/files/investigaciones/pib/departamentales/B_2005/Bol_dptal_2015preliminar.pdf

Departamento Administrativo Nacional de Estadísticas (DANE). (2017). Encuesta de Convivencia y Seguridad Ciudadana 2017. Recuperado de https://www.dane. gov.co/files/investigaciones/poblacion/convivencia/2017/Bol_ECSC_2017.pdf

Departamento Administrativo Nacional de Estadísticas (DANE). (2017). Medición de empleo informal y seguridad social. Recuperado de https://www.dane.gov.co/ files/investigaciones/boletines/ech/ech_informalidad/ bol_ech_informalidad_dic17_feb18.pdf

Espinosa Gutiérrez, C. M., Rodríguez Higuera, C. P. y Uribe Fernández, M. C. (2008). El impacto socioeconómico del mototaxismo en la ciudad de Monteria. (Tesis de grado) Universidad de la Sabana, Chía, Colombia. Recuperado de http://repositorio.unicartagena.edu.co:8080/jspui/bitstream/11227/2232/1/TESIS2015.pdf

Econometría Consultores S. A. (2013). Caracterización de la prestación del servicio de transporte en Motocicleta (Mototaxismo) en Colombia. Trabajo investigativo presentado a la Corporación Fondo de Prevención Vial.

García, I. y González, P. (2004). Características y determinantes del mercado del servicio de mototaxis en la ciudad de Cartagena. (Tesis de grado). Universidad de Cartagena, Cartagena, Colombia.

Glaeser, E. L. y Bruce, S. (1999). Why is There More Crime in Cities? Journal of Political Economy, 107(2), S225-S258.

Gómez Morris, S. y León Álvarez, W. (2015). Efectos económicos de la incidencia delictiva en la ciudad de Car- tagena durante el periodo 200-2012. (Tesis doctoral). Universidad de Cartagena, Cartagena, Colombia.

Instituto de Hidrología, Meteorología y Estudios Ambientales (Ideam) y Programa de las Naciones Unidas para el Desarrollo (PNUD). (2016). Inventario nacional $y$ departamental de Gases Efecto Invernadero-Colombia. Bogotá, Colombia: Zetta Comunicadores.

Organización Internacional del Trabajo (оIт). (2013a). La medición de la informalidad: manual estadístico sobre el sector informal y el empleo informal. Turín, Italia: Centro Internacional de Formación de la OIT.

Organización Internacional del Trabajo (OIT). (2013b). Resolución I: resolución sobre las estadísticas del trabajo, la ocupación y la subutilización de la fuerza de trabajo. En XIX Conferencia Internacional de Estadísticos del Trabajo. Ginebra, Organización Internacional del Trabajo (огт).

Presidencia de la República. (4 de septiembre de 2006). Decreto 2961. Bogotá, Colombia.

Programa de Las Naciones Unidas para el Desarrollo (PNUD) (2014). Estudio de perfiles ocupacionales para el mototaxismo en la ciudad de Valledupar. Bogotá, Colombia: Departamento para la Prosperidad Social (DPS).

Sánchez-Jabba, A. M. (2011). La economía del mototaxismo: el caso de Sincelejo. Documentos de Trabajo Sobre Economía Regional y Urbana, (140). Banco de la República.

Wooldridge, J. M. (2015). Introductory econometrics: A modern approach. Ciudad de México, México: Cengage Learning Editores. 


\section{Anexos}

Anexo 1. Estadísticas por ciudad y variable

\begin{tabular}{|c|c|c|c|c|c|c|c|c|c|c|}
\hline \multirow[b]{2}{*}{ Ciudad } & \multicolumn{2}{|c|}{ Hurto a personas } & \multicolumn{2}{|c|}{ Desempleo } & \multicolumn{2}{|c|}{ Inflación } & \multicolumn{2}{|c|}{ PIB Dptal. Constante } & \multicolumn{2}{|c|}{ Gini } \\
\hline & Media & $\begin{array}{l}\text { Desv. } \\
\text { Estándar }\end{array}$ & Media & $\begin{array}{l}\text { Desv. } \\
\text { estándar }\end{array}$ & Media & $\begin{array}{l}\text { Desv. } \\
\text { estándar }\end{array}$ & Media & $\begin{array}{l}\text { Desv. } \\
\text { estándar }\end{array}$ & Media & $\begin{array}{l}\text { Desv. } \\
\text { estándar }\end{array}$ \\
\hline
\end{tabular}

\begin{tabular}{|c|c|c|c|c|c|c|c|c|c|c|}
\hline Armenia & 8,72 & 0,74 & 14,5 & 0,26 & 3,92 & 0,94 & 7447,8 & 204,1 & 0,48 & 0,01 \\
\hline Bucaramanga & 6,02 & 0,58 & 7,9 & 0,17 & 4,26 & 0,61 & 39592,71 & 1105 & 0,42 & 0,01 \\
\hline Barranquilla & 6,88 & 0,77 & 8,05 & 0,14 & 4,39 & 0,93 & 33826,99 & 1114 & 0,45 & 0 \\
\hline Cúcuta & 6,87 & 0,51 & 13,92 & 0,6 & 3,25 & 0,93 & 13769,21 & 395,1 & 0,44 & 0 \\
\hline Cali & 9,2 & 0,79 & 12,12 & 0,37 & 3,95 & 0,8 & 69658,08 & 1833 & 0,49 & 0,01 \\
\hline Cartagena & 5,45 & 0,5 & 8,3 & 0,4 & 3,99 & 0,84 & 25938,76 & 908,8 & 0,47 & 0,01 \\
\hline Valledupar & 9,7 & 1,39 & 10,22 & 0,7 & 3,71 & 0,79 & 15366,78 & 468,1 & 0,46 & 0,01 \\
\hline Ibagué & 10,5 & 0,56 & 11,97 & 0,48 & 3,78 & 0,81 & 18334,31 & 377,6 & 0,44 & 0 \\
\hline Manizales & 9,27 & 0,73 & 10,27 & 0,3 & 4,36 & 0,99 & 12998,55 & 453,4 & 0,46 & 0 \\
\hline Medellín & 9,27 & 0,82 & 10,23 & 0,22 & 4,21 & 0,85 & 117324,62 & 3150 & 0,49 & 0,01 \\
\hline Neiva & 10 & 0,59 & 10,17 & 0,51 & 3,59 & 0,81 & 14004,35 & 224 & 0,47 & 0,01 \\
\hline Pasto & 20,7 & 1,33 & 10,02 & 0,65 & 3,84 & 0,96 & 12702,78 & 436,6 & 0,49 & 0,01 \\
\hline Pereira & 7,23 & 0,94 & 11,68 & 0,94 & 3,85 & 0,87 & 12517,77 & 345 & 0,44 & 0,01 \\
\hline Popayán & 12,1 & 0,67 & 11,42 & 0,75 & 3,82 & 0,92 & 12760,3 & 460,2 & 0,49 & 0 \\
\hline Santa Marta & 5,88 & 0,55 & 8,82 & 0,38 & 3,62 & 0,76 & 12245,9 & 189,2 & 0,46 & 0,01 \\
\hline Sincelejo & 6,97 & 0,28 & 9,33 & 0,1 & 3,88 & 1,09 & 6217,28 & 177,3 & 0,46 & 0,01 \\
\hline Villavicencio & 10,6 & 0,84 & 11,03 & 0,29 & 3,49 & 0,73 & 31792,99 & 540,7 & 0,45 & 0 \\
\hline
\end{tabular}

\begin{tabular}{|c|c|c|c|c|c|c|c|c|c|c|}
\hline \multirow{2}{*}{ Ciudad } & \multicolumn{2}{|c|}{ Índice de pobreza } & \multicolumn{2}{|c|}{$\begin{array}{l}\text { Población en } \\
\text { estrato bajo }\end{array}$} & \multicolumn{2}{|c|}{$\begin{array}{l}\text { Índice de } \\
\text { escolaridad }\end{array}$} & \multicolumn{2}{|c|}{$\begin{array}{l}\text { N. }{ }^{\circ} \text { cuadrantes de } \\
\text { pol/(1000)hab. }\end{array}$} & \multicolumn{2}{|c|}{$\begin{array}{c}\text { N. }{ }^{\circ} \text { CAI de pol/(1000) } \\
\text { hab. }\end{array}$} \\
\hline & Media & $\begin{array}{l}\text { Desv. } \\
\text { estándar }\end{array}$ & Media & $\begin{array}{l}\text { Desv. } \\
\text { estándar }\end{array}$ & Media & $\begin{array}{l}\text { Desv. } \\
\text { estándar }\end{array}$ & Media & $\begin{array}{l}\text { Desv. } \\
\text { estándar }\end{array}$ & Media & $\begin{array}{l}\text { Desv. } \\
\text { estándar }\end{array}$ \\
\hline Armenia & 26,5 & 1,38 & 57,35 & 1,2 & 9,47 & 0,06 & 1,38 & 0,27 & 4,4 & 0,02 \\
\hline Bucaramanga & 10,1 & 0,51 & 50,87 & 0,97 & 9,74 & 0,05 & 1,79 & 0,13 & 7,1 & 0,01 \\
\hline Barranquilla & 24,7 & 1,71 & 76,14 & 0,78 & 9,96 & 0,09 & 1,64 & 0,13 & 3 & 0 \\
\hline Cúcuta & 32,8 & 0,33 & 77,85 & 0,71 & 8,7 & 0,08 & 1,15 & 0,07 & 3 & 0,02 \\
\hline Cali & 18,6 & 1,36 & 55,73 & 0,55 & 9,59 & 0,05 & 1,32 & 0,09 & 1,7 & 0 \\
\hline Cartagena & 28,5 & 0,99 & 70,41 & 1,06 & 10,18 & 0,06 & 1,15 & 0,04 & 2,9 & 0 \\
\hline Valledupar & 31,7 & 1,21 & 76,73 & 0,81 & 9,9 & 0,08 & 0,88 & 0,26 & 3 & 0,01 \\
\hline Ibagué & 18,3 & 0,77 & 70,29 & 0,81 & 9,72 & 0,1 & 1 & 0,23 & 2,4 & 0 \\
\hline Manizales & 15,3 & 0,6 & 41,98 & 0,88 & 9,93 & 0,06 & 0,59 & 0,19 & 3,9 & 0,12 \\
\hline Medellín & 15,1 & 0,64 & 52,54 & 0,62 & 9,81 & 0,09 & 1,67 & 0,2 & 1,8 & 0 \\
\hline Neiva & 22,1 & 0,51 & 85,89 & 0,34 & 9,78 & 0,08 & 1,07 & 0,29 & 3 & 0,06 \\
\hline Pasto & 25,5 & 1,45 & 69,86 & 0,76 & 9,96 & 0,12 & 1,27 & 0,4 & 2,7 & 0,09 \\
\hline Pereira & 18,5 & 1,64 & 55,41 & 0,7 & 9,14 & 0,07 & 2,16 & 0,01 & 3,7 & 0,01 \\
\hline Popayán & 32,3 & 1,03 & 52,53 & 1,31 & 10,13 & 0,08 & 1,8 & 0,36 & 3 & 0,06 \\
\hline Santa Marta & 33,8 & 0,78 & 56,55 & 0,82 & 9,68 & 0,11 & 1,15 & 0,13 & 1 & 0 \\
\hline Sincelejo & 33,2 & 1,33 & 85,45 & 0,92 & 9,17 & 0,14 & 1,33 & 0,42 & 1,8 & 0 \\
\hline Villavicencio & 18,9 & 0,78 & 57,92 & 1,08 & 9,49 & 0,08 & 1,3 & 0,24 & 2,7 & 0 \\
\hline
\end{tabular}


Anexo 2. Prueba del multiplicador de Lagrange para efectos aleatorios de Breusch y Pagan

\section{Prueba del multiplicador de Lagrange para efectos aleatorios de Breusch y Pagan}

\begin{tabular}{cc} 
Chibar2(01) & 35,81 \\
\hline Prob>chibar2 & 0,0000 \\
\hline
\end{tabular}

Anexo 3. Prueba de Hausman

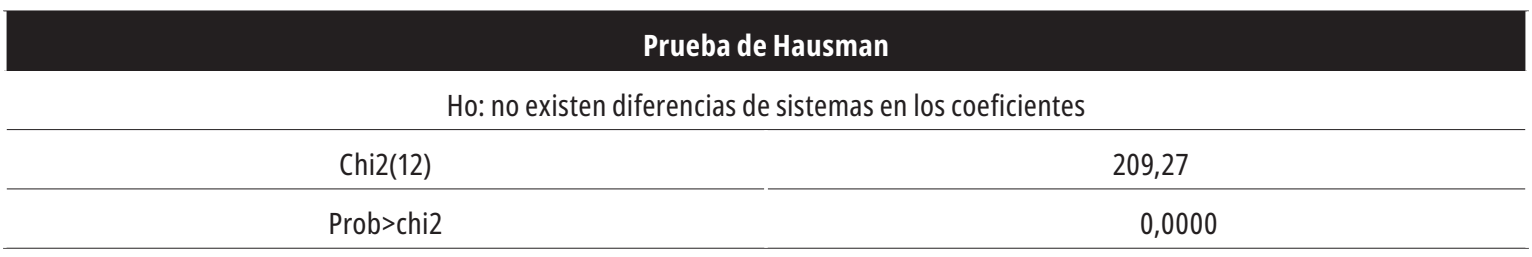

Anexo 4. Prueba de autocorrelación para panel de datos de Wooldridge

\begin{tabular}{ccc} 
& $\begin{array}{c}\text { Prueba de autocorrelación para panel de datos } \\
\text { de Wooldridge }\end{array}$ \\
\hline & Ho: no existe autocorrelación de primer orden \\
\hline $\mathrm{F}(1,16)$ & 1,33 \\
\hline $\mathrm{Prob}>\mathrm{F}$ & 0,2657 \\
\hline
\end{tabular}

Anexo 5. Prueba modificada de Wald para heteroscedasticidad

Prueba modificada de Wald para heteroscedasticidad para un modelo de efectos fijos

\begin{tabular}{cc}
\multicolumn{3}{c}{ Ho: $\operatorname{sigma}(\mathrm{i})^{\wedge} 2=\operatorname{sigma}{ }^{\wedge} 2$ para todo $\mathrm{i}$} \\
\hline Chi2(17) & 91,51 \\
\hline Prob>chi2 & 0,0000 \\
\hline
\end{tabular}

